\title{
Metaiodobenzylguanidine and Heart Rate Variability in Heart Failure
}

\author{
Chinori Kurata, MD; Sakae Shouda, MD; Tadashi Mikami, MD; Akihiko Uehara, MD; \\ Keiko Ishikawa, MT*; Kei Tawarahara, MD**; Tomoyasu Nakano, MD**; \\ Fumitaka Matoh, MD**; Kazuhiko Takeuchi, MD**
}

\begin{abstract}
It is assumed that the low-frequency power (LF) of heart rate variability (HRV) increases with progress of congestive heart failure (CHF), therefore positively correlating with cardiac ${ }^{123}$ I-metaiodobenzylguanidine (MIBG) washout. It is demonstrated here that HRV, including normalized LF, correlated inversely with MIBG washout and positively with the ratio of heart-to-mediastinum MIBG activity in controls and CHF patients, whereas these correlations were not observed within CHF patients. Thus MIBG washout may increase and HRV including normalized LF may decrease with CHF, although the HRV and MIBG measures may not similarly change in proportion to the severity of the cardiac autonomic dysfunction in CHF. (Jpn Circ J 1998; 62: 770772)
\end{abstract}

Key Words: Congestive heart failure; ${ }^{123}$ I-metaiodobenzylguanidine; Heart rate variability

$\mathbf{T}$ he cardiac sympathetic nerve system has been evaluated with the low-frequency power (LF) of heart rate variability (HRV) ${ }^{1}$ and cardiac ${ }^{123} \mathrm{I}_{-}$ metaiodobenzylguanidine (MIBG) washout? Recently, Somsen et al reported that the cardiac MIBG washout rate positively correlated with normalized LF in mild to moderate congestive heart failure (CHF), which is contrary to our earlier finding, ${ }^{4}$ that is, an inverse correlation of the MIBG washout rate with HRV including LF in various disorders. Furthermore, the normalized LF of their CHF patients $[34.9 \pm 7 \%$, mean $(\mathrm{SD})]$ was lower than normal values $(54 \pm 4 \%)$ despite a similar MIBG washout rate $(7.7 \pm 8.4 \%$ vs $6.5 \pm 5.3 \%)$ ? We therefore re-examined the correlation between MIBG and HRV in CHF.

\section{Methods}

The patient group comprised 20 stable CHF patients (age, $49 \pm 15$ years; female/male, 6/14) who underwent MIBG scintigraphy and 24-h ambulatory electrocardiography within 1 week and who had neither diabetes mellitus nor frequent arrhythmia. The control group comprised 11 age- and gender-matched normal subjects. The following MIBG and HRV studies were performed as a routine clinical examination in the patient group, while informed consent was obtained in the control group.

On the early and late anterior images obtained $15 \mathrm{~min}$ and 150 min after MIBG injection, respectively, mean MIBG activities per pixel were measured over the heart $(\mathrm{H})$, background $(\mathrm{BG})$ around the heart, and upper mediastinum (M) $)^{2,4}$ Heart-to-mediastinum activity ratio (H/M)

(Received May 13, 1998; revised manuscript received July 3, 1998; accepted July 24, 1998)

Departments of Medicine III and *Clinical Laboratories, Hamamatsu University School of Medicine, and **Department of Internal Medicine, Hamamatsu Red Cross Hospital, Hamamatsu, Japan

Mailing address: Chinori Kurata, Department of Medicine III, Hamamatsu University School of Medicine, 3600 Handa-cho, Hamamatsu 431-3192, Japan. E-mail: kurata-c@ @ost.yamaha.co.jp was calculated in the early and late images, and the cardiac MIBG washout rate (\%) was calculated with the formula:

$$
\begin{aligned}
([\text { early } \mathrm{H} \text {-early BG] } & -[\text { late } \mathrm{H}-\text { late BG] }) \\
& \times 100 /[\text { early } \mathrm{H}-\text { early BG]. }
\end{aligned}
$$

Blood samples were obtained via an indwelling catheter in the forearm vein to measure plasma norepinephrine (pNE) levels, immediately before MIBG administration (from 9.00 to $11.00 \mathrm{~h}$ ), while the patients were lying at rest. The pNE levels $(\mathrm{pg} / \mathrm{ml})$ were determined by high-performance liquid chromatography.

Ambulatory 24-h electrocardiograms were recorded; the time series of normal RR intervals for $24 \mathrm{~h}$ was analyzed with a fast Fourier transform algorithm; and LF (0.04-0.15 $\mathrm{Hz}$ ), high-frequency $(\mathrm{HF})$ power $(0.15-0.40 \mathrm{~Hz}$,$) , total$ power (TP), and normalized LF (LF/TP) were computed using a Marquette HRV program. We also calculated 2 time-domain measures: 24-h SD of normal RR intervals $(\mathrm{SDNN})$ and the percentage of differences between adjacent normal RR intervals $>50 \mathrm{msec}$ (pNN50).

All measures were expressed as mean \pm SD. Differences between groups were examined by the Student's t-test.

Table 1 Comparisons Between Controls and Patients

\begin{tabular}{lccc}
\hline \hline \multicolumn{1}{c}{ Parameters } & Controls & Patients & $P$ \\
\hline MIBG washout $(\%)$ & $-1 \pm 12$ & $45 \pm 25$ & $<0.001$ \\
Early H/M & $2.18 \pm 0.30$ & $1.64 \pm 0.19$ & $<0.001$ \\
Late H/M & $2.42 \pm 0.26$ & $1.49 \pm 0.19$ & $<0.001$ \\
In[TP] $\left(\right.$ ln $\left.\left[\mathrm{msec}^{2}\right]\right)$ & $7.45 \pm 0.53$ & $5.62 \pm 1.14$ & $<0.001$ \\
ln[LF] $\left(\right.$ ln $\left.\left[\mathrm{msec}^{2}\right]\right)$ & $6.56 \pm 0.59$ & $4.08 \pm 1.45$ & $<0.001$ \\
ln[HF] $\left(\ln \left[\mathrm{msec}^{2}\right]\right)$ & $5.38 \pm 0.68$ & $3.31 \pm 1.30$ & $<0.001$ \\
LF/TP & $0.41 \pm 0.05$ & $0.23 \pm 0.08$ & $<0.001$ \\
SDNN $(\mathrm{msec})$ & $159 \pm 25$ & $90 \pm 40$ & $<0.001$ \\
pNN50 $(\%)$ & $12 \pm 9$ & $3 \pm 4$ & 0.010 \\
\hline
\end{tabular}

$H / M$, heart-to-mediastinum activity ratio; TP, total power; LF, lowfrequency power; HF, high-frequency power; SDNN, 24-h SD of normal $R R$ intervals; pNN50, percentage of differences between adjacent normal $R R$ intervals $>50 \mathrm{msec}$. 
Correlations were expressed as Pearson correlation coefficients. Logarithm transformation was used before analysis of some variables to make them normally distributed. Statistical significance was defined as $\mathrm{p}<0.05$.

\section{Results}

Table 1 shows the comparison between controls and CHF patients. The cardiac MIBG washout rate was significantly higher, early and late $\mathrm{H} / \mathrm{M}$ were significantly lower, and all HRV measures including LF/TP were significantly lower in the 20 patients than in the 11 controls. In the total 31 subjects, the MIBG washout rate correlated inversely with SDNN ( $\mathrm{r}=-0.57, \mathrm{p}<0.001), \ln [\mathrm{TP}], \ln [\mathrm{LF}], \mathrm{LF} / \mathrm{TP}$ $(\mathrm{r}=-0.45,-0.47,-0.54 ; \mathrm{p}<0.01 ; \mathrm{Fig} 1), \ln [\mathrm{HF}]$ and $\mathrm{pNN} 50$ $(\mathrm{r}=-0.41,-0.38 ; \mathrm{p}<0.05)$. The early $\mathrm{H} / \mathrm{M}$ correlated with $\ln [\mathrm{TP}], \ln [\mathrm{LF}], \ln [\mathrm{HF}], \mathrm{LF} / \mathrm{TP}, \mathrm{pNN} 50$ ( $\mathrm{r}=0.46,0.48,0.50$, $0.55,0.53 ; \mathrm{p}<0.01)$ and SDNN $(\mathrm{r}=0.42, \mathrm{p}<0.05)$. The late $\mathrm{H} / \mathrm{M}$ also correlated with $\ln [\mathrm{TP}], \ln [\mathrm{LF}], \ln [\mathrm{HF}], \mathrm{LF} / \mathrm{TP}$, $\mathrm{SDNN}$, and pNN50 (r=0.60, 0.63, 0.62, 0.70, 0.65, 0.63; $\mathrm{p}<0.001$; Fig 1). Within the 20 CHF patients, however, there was no significant correlation between the MIBG and HRV measures. On the other hand, within the 11 control subjects, the early $\mathrm{H} / \mathrm{M}$ correlated with $\ln [\mathrm{HF}]$ and the late

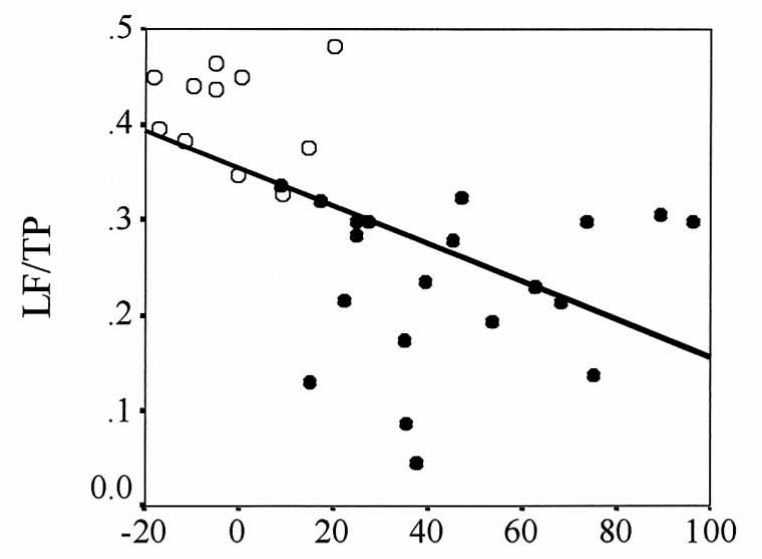

\section{Cardiac MIBG washout (\%)}

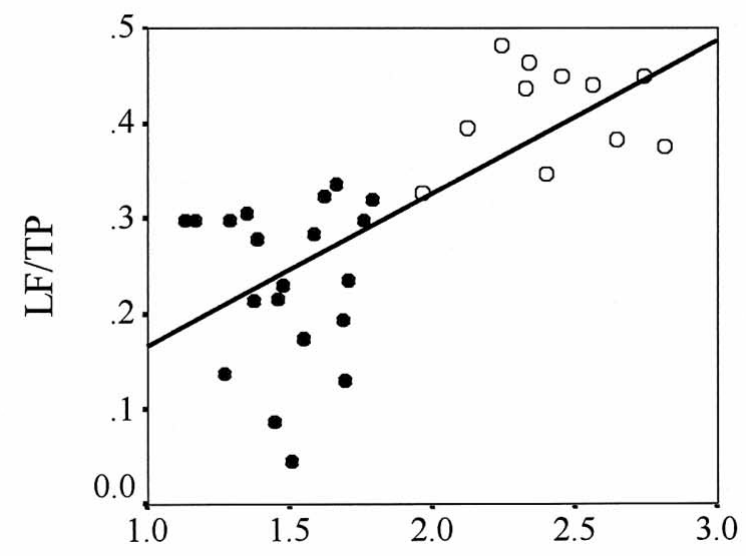

\section{Late $\mathrm{H} / \mathrm{M}$}

Fig 1. Relation between cardiac MIBG washout rate and LF/TP (top: $\mathrm{r}=-0.54, \mathrm{p}<0.01$ ) and between late $\mathrm{H} / \mathrm{M}$ and $\mathrm{LF} / \mathrm{TP}$ (bottom: $\mathrm{r}=0.70, \mathrm{p}<0.001)$. (Open circle) Controls, (closed circle) patients.
$\mathrm{H} / \mathrm{M}$ correlated with $\ln [\mathrm{HF}]$ and $\mathrm{pNN} 50$ ( $\mathrm{r}=0.70,0.67$, $0.71 ; \mathrm{p}<0.05)$, but no other significant correlation was found between the MIBG and HRV measures.

In the total 31 subjects, the pNE level correlated with the MIBG washout rate $(\mathrm{r}=0.55, \mathrm{p}<0.01)$ and inversely with late $\mathrm{H} / \mathrm{M}, \ln [\mathrm{TP}], \ln [\mathrm{LF}], \mathrm{LF} / \mathrm{TP}(\mathrm{r}=-0.55,-0.54,-0.47$, $-0.49 ; \mathrm{p}<0.01)$, early $\mathrm{H} / \mathrm{M}$ and $\ln [\mathrm{HF}](\mathrm{r}=-0.45,-0.43$; $\mathrm{p}<0.05$ ). Within the $20 \mathrm{CHF}$ patients, however, there was no significant correlation of $\mathrm{pNE}$ with the MIBG and HRV measures.

\section{Discussion}

We demonstrated enhanced cardiac MIBG washout and diminished HRV including LF and LF/TP in CHF patients, which is compatible with earlier reports ${ }^{5-8}$ The absence of significant correlation between LF/TP and cardiac MIBG washout in our CHF patients, however, is not consistent with the results of Somsen et al3 which may be due to several differences between our and their studies. First, background subtraction was applied to the calculation of MIBG washout rate in our study but not in theirs. Second, $\mathrm{pNE}$ was not increased in all their patients $(276 \pm 84 \mathrm{pg} / \mathrm{ml})$ but was high in some of ours $(63-3130,792 \pm 640 \mathrm{pg} / \mathrm{ml})$. However, these differences may not be significant, because there was no correlation $(r=0.04 ; p=0.92)$ between LF/TP and the MIBG washout rate without background subtraction in our 8 patients without increased pNE. Third, a difference in severity of CHF, which was suggested by the difference in $\mathrm{pNE}$ level, may be involved in the presence or absence of a correlation between LF/TP and the cardiac MIBG washout, because LF may be markedly diminished in patients with severe CHF but not in those with mild CHF ${ }^{6,8}$ Lastly, the positive correlation observed by Somsen et $\mathrm{al}^{3}$ depended on only 2 of 10 patients and, therefore, might be purely coincidental.

In CHF, as shown in previous studies, ${ }^{3,5-9} \mathrm{HRV}$ including LF/TP may decrease while cardiac MIBG washout rate may increase. An inverse correlation is therefore inferred between the LF/TP and MIBG washout rate in the controls and $\mathrm{CHF}$ patients. In contrast, we showed a lack of correlation between the MIBG washout rate and HRV including LF/TP within the CHF patients, suggesting that HRV and MIBG measures may not similarly change in proportion to the severity of cardiac autonomic dysfunction in CHF, 68 Moreover, the LF (or LF/TP) of HRV may not be an appropriate measure of cardiac sympathetic nerve activity, although it may have prognostic value in CHF patients. Thus, using MIBG we can approach cardiac sympathetic nerve system abnormalities in CHF from a different aspect than HRV.

\section{References}

1. Task Force of European Society of Cardiology and North American Society of Pacing and Electrophysiology: Heart rate variability: standards and measurement, physiological interpretation, and clinical use. Circulation 1996; 93: 1043-1065

2. Kurata C, Wakabayashi Y, Shouda S, Okayama K, Yamamoto T, Ishikawa A, et al: Enhanced clearance of 123I-metaiodobenzylguanidine from the heart in patients with chronic renal failure on dialysis. J Nucl Med 1995; 36: 2037-2043

3. Somsen GA, Szabó BM, van Veldhuisen DJ, de Milliano PAR, de Groot CA, Lie KI: Comparison between iodine 123-metaiodobenzylguanidine scintigraphy and heart rate variability for the assessment of cardiac sympathetic activity in mild to moderate heart failure. Am Heart J 1997; 134: 456-458

4. Kurata C, Shouda S, Mikami T, Wakabayashi Y, Nakano T, 
Sugiyama T, et al: Comparison of ${ }^{123}$ I-metaiodobenzylguanidine kinetics with heart rate variability and plasma norepinephrine. $J \mathrm{NuCl}$ Cardiol 1997; 4: 515-523

5. Henderson EB, Kahn JK, Corbett JR, Jansen DE, Pippin JJ, Kulkarni $\mathrm{P}$, et al: Abnormal I-123 metaiodobenzylguanidine myocardial washout and distribution may reflect myocardial adrenergic derangement in patients with congestive cardiomyopathy. Circulation 1988; 78: $1192-1199$

6. Van de Borne P, Montano N, Pagani M, Oren R, Somers VK: Absence of low-frequency variability of sympathetic nerve activity in severe heart failure. Circulation 1997; 95: 1449-1454

7. Shiigi T, Furutani Y, Nakamura Y, Nakamura H, Harada M, Yamamoto T, et al: Relative usefulness of measures of heart rate variability and neuroendocrine activity as indicators of autonomic nervous abnormality in patients with left ventricular dysfunction. Jpn Circ J 1998; 62: 336-340

8. Mortara A, La Rovere MT, Signorini MG, Pantaleo P, Pinna G, Martinelli L, et al: Can power spectral analysis of heart rate variability identify a high risk subgroup of congestive heart failure patients with excessive sympathetic activation? A pilot study before and after heart transplantation. Br Heart J 1994; 71: 422-430

9. Kingwell BA, Thompson JM, Kaye DM, McPherson GA, Jennings GL, Esler MD: Heart rate spectral analysis, cardiac norepinephrine spillover, and muscle sympathetic nerve activity during human sympathetic nervous activation and failure. Circulation 1994; 90: $234-240$ 